\title{
Documentation and treatment of hypertension: quality of care and missed opportunities in a family medicine resident clinic
}

\author{
Phillip So, ${ }^{1}$ Steven Y Lin ${ }^{2}$
}

${ }^{1}$ Wayne State University School of Medicine, Detroit, Michigan, USA

${ }^{2}$ Division of General Medical Disciplines, Department of Medicine, Center for Education and Research in Family and Community Medicine, Stanford University School of Medicine, Stanford, California, USA

\section{Correspondence to} Phillip So, Wayne State University School of Medicine, 540 East Canfield Street, Detroit, MI 48201, USA phillip.cj.so@gmail.com

Received 6 December 2013 Revised 1 December 2014 Accepted 2 December 2014

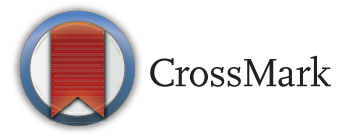

To cite: So P, Lin SY. Postgrad Med J

2015;91:30-34.

\section{ABSTRACT}

Background In the USA, uncontrolled hypertension contributes to 1000 deaths a day. However, little is known about the quality of hypertension management in family medicine resident clinics.

Objectives To examine rates of documentation and treatment of elevated blood pressure, and to identify missed opportunities to address hypertension.

Study design A cross-sectional chart review of 1011 adult patient visits between 2 January and 4 February 2013 was performed in a resident-run federally qualified health centre. For patients with elevated blood pressure at time of visit, we noted whether or not the residents had documented a diagnosis or discussion of hypertension and the presence or absence of a treatment plan. We compared these rates with those from a national sample of primary care physicians.

Results 262/1011 (26\%) of adult patients had elevated blood pressure at time of visit. Of those, 115/262 (44\%) had documentation and a plan for treatment, 79/262 (30\%) had documentation but no plan, and 68/262 (26\%) had neither documentation nor plan. Nationally, $45 \%$ of patients are diagnosed and treated compared with $44 \%$ of study visits with documentation and treatment.

Conclusions Fewer than half of visits of patients with elevated blood pressure resulted in both documentation and a treatment plan. Nevertheless, these rates are comparable to national providers. Elevated blood pressure was more likely to be missed during acute visits and in patients with less elevated blood pressure.

\section{INTRODUCTION}

In 2012, the Centers for Disease Control and Prevention (CDC) named hypertension 'public health enemy number two' behind only tobacco as the most deadly disease-causing condition. ${ }^{1}$ Nearly one-third of adults in the USA have hypertension, and more than half are uncontrolled. ${ }^{2}$ Uncontrolled hypertension contributes to 1000 deaths a day and US\$131 billion a year in healthcare costs. ${ }^{3-5}$ It is a significant cause of morbidity and mortality, including but not limited to heart attacks, strokes, heart failure and chronic kidney disease. $^{5}{ }^{6}$ According to the National Health and Nutrition Examination Survey (NHANES), ${ }^{7}$ of the 36 million Americans with uncontrolled hypertension, 26 million have seen a doctor at least twice in the previous year and nearly $90 \%$ have a usual source of healthcare and health insurance. ${ }^{8}$ These statistics suggest that many patient-physician encounters are missed opportunities for hypertension control.

One possible way to reduce the number of missed opportunities is to ensure that primary care physicians in training understand the importance of documenting elevated blood pressure and initiating a follow-up or treatment plan at every visit. Previous studies on the quality of hypertension care in resident clinics have focused on meeting treatment goals in patients with diagnosed hypertension. ${ }^{9}{ }^{10}$ To the best of our knowledge, there are no studies that have examined how often residents diagnose, discuss or treat elevated blood pressure at time of visit. The goals of this cross-sectional study were to: (1) assess the quality of hypertension management by family medicine residents using documentation of hypertension diagnosis or discussion and presence of treatment plans as outcome measures; (2) identify missed opportunities by investigating differences in documentation and treatment by visit type and severity of hypertension; (3) compare family medicine resident documentation and treatment rates with those of a national survey of primary care physicians.

\section{METHODS}

\section{Study setting}

The setting for this study was the Family Health Center at O'Connor Hospital, a federally qualified health centre that provides primary and urgent care to a predominantly low-income, underinsured (mostly Medicaid), ethnically diverse (largely Hispanic and Vietnamese) population in San Jose, California. The clinic is staffed by 24 family medicine residents from the San Jose-O'Connor Family Medicine Residency Program, affiliated with Stanford University School of Medicine. Each resident is individually responsible for his or her own panel of patients. At least two members of the faculty are always available for supervision and consultation. First-year residents are required to present every patient to a faculty member, while second- and third-year residents are encouraged, but not required, to do so.

There are two types of visits available at the Family Health Center. Patients with urgent concerns are able to request acute visits, which are 15 min long and are scheduled with a rotating resident assigned to clinic each day. In comparison, routine visits are typically scheduled with a resident who is the patient's primary care physician. These visits can last from 15 to $30 \mathrm{~min}$. During each visit, a medical assistant or nurse records blood pressure and other vital signs before the patient is seen. This information is available to the resident during the visit via the electronic medical record. All visits are performed directly by the residents with attending supervision. 


\section{Chart review}

One of the authors (SYL) abstracted data from the electronic medical records of all adult patient visits at the Family Health Center between 2 January 2013 and 4 February 2013. We excluded visits for children, adolescents (under 18 years of age), and pregnant women as well as visits during which no blood pressure was recorded (eg, nursing visits, educational or counselling sessions, and wheelchair users).

\section{Categorisation of elevated blood pressure}

We considered patients to have elevated blood pressure if their systolic or diastolic blood pressure was greater than 140 or $90 \mathrm{~mm} \mathrm{Hg}$, respectively, at time of visit. ${ }^{3}$ We further categorised them as meeting criteria for stage 1 (140-159/90-99 mm Hg), stage $2(160-179 / 100-110 \mathrm{~mm} \mathrm{Hg})$ or stage $3(180+/ 110+$ $\mathrm{mm} \mathrm{Hg}$ ) hypertension, according to Joint National Committee definitions. ${ }^{3}$ It is important to note that we categorised visits into stages of hypertension based on one reading and therefore these patients did not necessarily have or merit an official diagnosis of hypertension.

In order to gain more insight about patients with the highest blood pressures, we investigated visits with stage 3 blood pressure for any associations between factors such as age, body mass index (BMI), sex, diabetes, chief complaint, type of visit, and treatment plan. In addition, we examined each member of this cohort with regard to how his or her blood pressure was managed at the Family Health Center up to 4 months before and after the study timeline.

\section{Documentation and treatment}

The study included visits with elevated blood pressure regardless of chief complaint. We categorised visits by the presence or absence of blood pressure documentation, whether the visit type was acute or routine, whether a follow-up or treatment plan was documented, and the specifics of the plan (ie, medication, monitoring, lifestyle modifications, laboratory testing, or referral). Although all patients had their blood pressure recorded for each visit by a medical assistant or nurse, elevated blood pressure was only considered to be 'documented' if mentioned in the resident's note either via discussion in plain text or as a diagnosis code. Treatment was defined as the presence of a documented plan of action, which could include monitoring without any other intervention.

\section{Data analysis}

We divided patients with blood pressure of $140 / 90 \mathrm{~mm} \mathrm{Hg}$ or higher at time of visit into three categories: those whose blood pressure was documented in the resident's note (either via discussion in plain text or as a diagnosis code), those whose blood pressure was documented along with a plan for follow-up or treatment, and those without mention of either the elevated blood pressure or a treatment plan. We analysed differences in rates of documentation and presence of treatment plan by visit type and stage of hypertension. Rates were compared with analogous statistics from the NHANES ${ }^{7}$ in order to compare resident quality of care with those of a national sample of physicians.

\section{Comparison with national providers}

The results of this study were compared with those of the NHANES, a nationwide programme that surveys the health and nutritional status of a representative sample of Americans. It is carried out by physicians and health staff in participants' homes and in mobile health centres. The NHANES records medical diagnoses of hypertension as well as whether the participant is aware of their hypertension and whether they are under treatment by a physician. ${ }^{7}$ We compared study data with NHANES data as follows: rates of elevated blood pressure during visits were compared with rates of diagnosed hypertension; documentation of blood pressure by residents was related to patient awareness of their hypertension; initiation of treatment plan was related to rates of treatment by physicians.

\section{Statistical analysis}

Data were summarised using mean \pm SD or percentage. The $\chi^{2}$ test was used to compare proportions between groups. $\mathrm{p}$ Values for significance were indicated where relevant.

\section{Institutional review board}

The study protocol was reviewed by the institutional review board of O'Connor Hospital and granted exemption.

\section{RESULTS}

\section{Patient documentation and treatment}

Of the 1011 patient visits studied, 262 (26\%) included blood pressure readings in the hypertensive range $(>140 / 90)$. Of those, fewer than half $(115 / 262$ or $44 \%)$ had a documented discussion or diagnosis of hypertension and a plan for follow-up or treatment plan, 79/262 (30\%) had documentation of elevated blood pressure but no follow-up or treatment plan, and 68/262 (26\%) had neither documentation nor plan (figure 1).

\section{Management by hypertensive stage}

Of the 262 visits with hypertensive patients, 187/262 (71\%) were in the stage 1 range, 45/262 (17\%) were stage 2, and 30/ $262(12 \%)$ were stage 3 . Among stage 1 hypertensive patients, documentation and treatment status was divided approximately equally into all three documentation categories: 65/187 (35\%) visits had a documented discussion or diagnosis regarding hypertension and a treatment plan, 62/187 (36\%) visits had documentation without a plan, and 60/187 (32\%) visits had neither documentation nor plan. The proportion of patients with both documentation and a treatment plan increased for stage 2 and stage 3 hypertensive patients $(53 \%$ and $87 \%$, respectively) (figure 2). These increases were significant

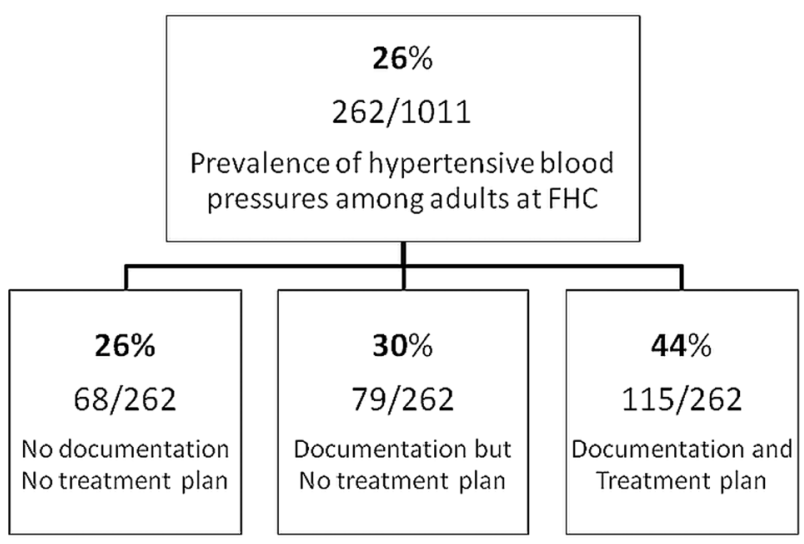

Figure 1 Classification of hypertensive patients by documentation and treatment. The study included 1011 consecutive visits, of which 262 featured hypertensive patients. The 262 hypertensive visits were classified by whether high blood pressure was undocumented, documented without a treatment plan, or documented with a treatment plan. FHC, Family Health Center. 


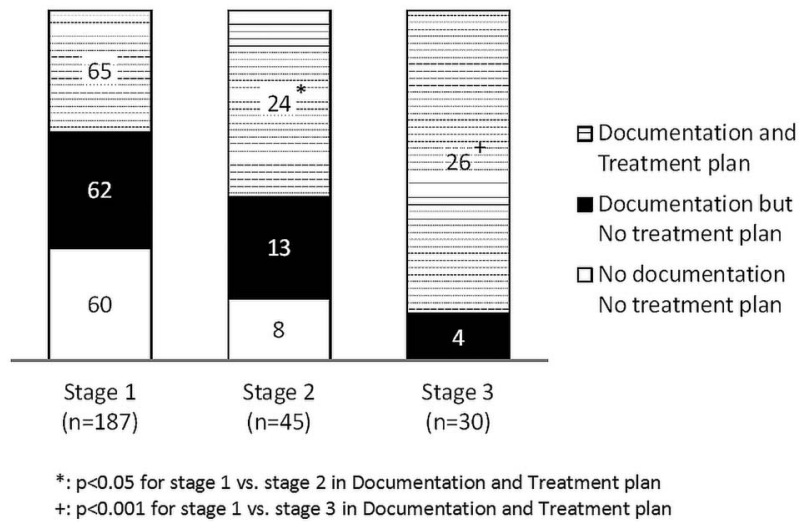

Figure 2 Hypertensive management by stage. The study included 262 visits with hypertensive patients. These visits were further divided in stage 1, 2 and 3 hypertensive patients (stage 1, 140-159/9099 mm Hg; stage 2, 160-179/100-110 mm Hg; stage 3, 180+/110+ $\mathrm{mm} \mathrm{Hg}$ ). Classification of the visits by documentation/treatment level and stage of hypertension is shown. Differences between the proportion of patients who were documented and treated in stage 1 compared with stage 2 and 3 were significant $(p<0.05$ and $p<0.001)$.

$(\mathrm{p}<0.05$ and $\mathrm{p}<0.001$, respectively). Other differences between stages were not significant.

\section{Management by visit type}

Of the 262 visits with hypertensive patients, 186/262 (71\%) were scheduled as routine and 76/262 (29\%) were scheduled as acute. Among routine visits, 98/186 (53\%) resulted in both documentation of blood pressure discussion or diagnosis and a treatment plan, 51/186 (27\%) visits had documentation but no plan, and 37/186 (20\%) had neither documentation nor plan. In contrast, among acute visits, only $17 / 76$ (22\%) had both documentation and a treatment plan, 28/76 (37\%) had documentation but no plan, and 31/76 (41\%) had neither documentation nor plan (figure 3). The difference between the number of acute and routine visits with both documentation and a treatment plan was significant $(\mathrm{p}<0.001)$.

\section{Treatment plans}

Of the patients who were prescribed a treatment plan, 48/115 (42\%) received medication management, 46/115 (40\%) were assigned home blood pressure monitoring without starting a

\section{$\square$ Acute visits ( $n=76) \quad$ Routine visits $(n=186)$}

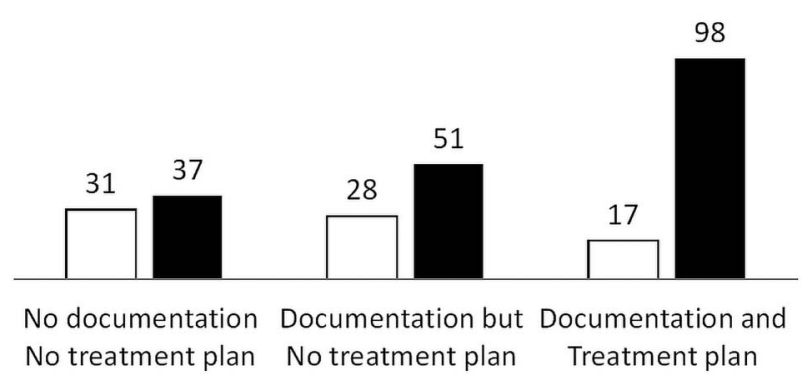

Figure 3 Classification of hypertensive patients by documentation and visit type. The study included 262 visits with hypertensive patients. Visits were either acute (shorter appointment, patient seen by rotating resident) or routine (longer appointment, patient seen by his/her primary care resident). Classification of the visits by documentation/ treatment level and visit type is shown. new medication, 9/115 (8\%) were referred for laboratory workup of secondary causes of hypertension, 9/115 (8\%) were referred to another department (eg, nephrology, cardiology, emergency room), and 3/115 (2\%) were given lifestyle (ie, diet and exercise) counselling without pharmacological intervention.

\section{Comparison with NHANES}

The prevalence of elevated blood pressure in the study population was $26 \%$, compared with the national prevalence of $16 \%$ estimated by 2010 NHANES data. ${ }^{8}$ The proportion of visits with elevated blood pressure and both documentation (discussion or diagnosis) and a treatment plan was 44\%, compared with the $45 \%$ of hypertension patients nationally who were aware of their status and were being treated. ${ }^{8}$ The proportion of visits with elevated blood pressure and documentation but no treatment plan was $30 \%$, compared with the $16 \%$ of hypertension patients nationally who were aware of their status but are not being treated. ${ }^{8}$ Finally, the proportion of visits with elevated blood pressure and no documentation or treatment plan was $26 \%$, compared with the $39 \%$ of hypertension patients nationally who were unaware of their high blood pressure and were not being treated. ${ }^{8}$

\section{Stage 3 hypertensive patients}

The 30 visits with patients who presented with blood pressure readings in the stage 3 range represented 24 unique individuals. One patient had three separate visits, and four other patients had two visits each during the 1 month study period. Most of the stage 3 hypertensive patients were female (13/24 or $54 \%$ ). The mean age was $52 \pm 18$ years. The mean BMI was $36 \pm 9$. Seven (29\%) patients had a diagnosis of type 2 diabetes mellitus. One in five of these visits were for acute concerns. In this small sample, no significant associations between sex, age, BMI, diabetes status, and type of treatment were found. Of the 30 visits with stage 3 hypertensive patients, $26(87 \%)$ resulted in a documented treatment plan, but four (13\%) did not. Most treatment plans for these patients $(16 / 26$ or $62 \%)$ included medication management.

\section{DISCUSSION}

As hypertension continues to represent a large burden of disease in the USA, training physicians must learn more than how to manage hypertension. They must also grow accustomed to addressing hypertension at every visit by discussing or diagnosing newly elevated blood pressure and ensuring appropriate treatment. For more than half of patients with elevated blood pressure at time of visit, a documented diagnosis or discussion of hypertension, a plan for treatment, or both was missing. Residents were more than twice as likely to document elevated blood pressure and initiate treatment plans during routine visits compared with acute visits (53\% vs 22\%). Stage 1 and 2 hypertensive patients were less likely to have their elevated blood pressures documented and less likely to receive a treatment plan compared with stage 3 hypertensive patients (35\% and 53\% vs $87 \%)$. Therefore, patients seen in acute care visits and those with mild to moderately elevated (stage 1 and 2) blood pressure represent important sources of missed opportunities for care.

Elevated blood pressure may have been neglected during acute visits for many reasons. Patients at an acute visit were usually seen by randomly assigned residents who may tend to defer management of chronic conditions such as hypertension to their colleagues who are the patients' primary care resident. However, without clear documentation, repeated elevations in blood pressure during acute visits may never be seen or 
addressed by the primary care resident. Furthermore, patients at an acute visit often sought care for urgent complaints unrelated to hypertension and were limited to $15 \mathrm{~min}$ appointments. It is possible that residents chose to prioritise the patients' concerns and defer hypertension management, thus missing an opportunity to address elevated blood pressure. Visits with only mild to moderately elevated (stage 1 and 2) blood pressure may be even more susceptible to becoming missed opportunities.

Family medicine residents recognised and treated elevated blood pressure at a similar rate to a national survey of primary care physicians in the NHANES (44\% vs $45 \%$ ). Therefore, in terms of these basic quality measures of hypertension management, it appears that family medicine residents perform comparably to the national population of providers. However, the proportion of visits with documentation of hypertension diagnosis or discussion but no treatment plan was almost double $(30 \%$ vs $16 \%$ ) the proportion of patients nationally who were aware of their hypertension but not treated. This suggests that, while family medicine residents may adequately recognise elevated blood pressure, they may not be initiating plans for follow-up or treatment. This could be due to many factors such as a relative lack of confidence and experience in choosing the best treatment regimen and fear of non-compliance. The proportion of visits with elevated blood pressure that resulted in no documentation or treatment was lower (26\% vs $39 \%$ ) than the proportion of hypertension patients nationally who were unaware of their hypertension and were not being treated. ${ }^{8}$ Although the rate of documenting without treatment is higher for residents, the comparable proportion of visits with documentation and treatment and the lower proportion of patients without documentation or treatment suggests that family medicine residents perform comparably to a national survey of providers. These findings are in general agreement with other studies that have demonstrated the ability of residents to deliver high-quality care. ${ }^{9} 10$

When a plan to address an elevated blood pressure was established, residents chose to prescribe further blood pressure monitoring as often as they prescribed medications. The implications of this finding are unclear. Holding treatment may be related to an uncertain diagnosis (lack of repeated abnormal blood pressure readings) or confounding factors (eg, white coat hypertension, transiently elevated blood pressure due to pain). In addition, residents may be concerned about a lack of time to initiate antihypertensive therapy in the setting of multiple patient complaints, especially during acute visits. While home blood pressure monitoring may be a valid plan for many patients (especially those with borderline hypertension), examination of stage 3 hypertensive patients suggested that as many as half of these patients would not receive appropriate treatment during follow-up visits over the subsequent months despite persistently elevated blood pressure. This suggests that every missed opportunity to initiate treatment increases the risk of patients falling through the cracks and being lost to follow-up.

This study has several limitations. Its cross-sectional nature examines documentation of discussion, diagnosis and treatment of elevated blood pressure at a single visit, which may not be representative of overall management of hypertension over time. Blood pressure readings were not averaged over several readings, so it is possible that some recordings were increased artificially (error in measurements, white coat hypertension, etc) and do not require management. However, this study's focus was identifying rates of documentation and missed opportunities, rather than evaluating chronic management of diagnosed hypertension. It should be noted that this chart review analysed individual visits and it is possible that some visits involved the same patient, although this is unlikely given the short timeline.
The NHANES was used to compare the performance of family medicine residents with a national sample of physicians. However, there are important differences between the study data and the NHANES. This study focused on resident-reported documentation and initiation of treatment plans. The NHANES data included objective blood pressure measurements as well as patient-reported awareness and treatment status. Patient responses may not necessarily have corresponded to their doctors' assessments or plans. The NHANES is also designed to be a representative sample of the entire American population and their physicians, while our study focused on low-income patients at a resident-run federally qualified health centre. Resources available to physicians, patient compliance, insurance coverage, and other socioeconomic factors are likely to differ. The comparable performance of family medicine residents in the context of low-income patients in a federally funded clinic is a promising sign.

On the other hand, it is unclear whether the results of this study can be generalised to family medicine residents at suburban or rural programmes with different patient populations.

\section{Main messages}

- Residents working in family medicine often fail to focus on management of raised blood pressure in their clinic patients.

- Residents were especially unlikely to address or treat elevated blood pressure during acute visits and in patients with less elevated blood pressure.

- Family medicine residents diagnose, discuss and treat elevated blood pressure at similar rates to a national survey of primary care physicians.

\section{Current research questions}

- What do physicians and patients report as barriers to addressing hypertension?

- What kinds of interventions might circumvent these barriers?

- How can patients be encouraged to prioritise blood pressure and schedule routine visits for hypertension management?

\section{Key references}

- CDC. National Health and Nutrition Examination Survey: surveys and data collection systems (2010 data). Atlanta, GA: US Department of Health and Human Services, CDC, National Center for Health Statistics; 2012. Available at http://www.cdc.gov/nchs/nhanes.htm (accessed 20 June 2013).

- CDC. Vital signs: awareness and treatment of uncontrolled hypertension among adults-United States, 2003-2010. MMWR 2012;61:703-9.

- Masterson E, Patel P, Kuo Y, et al. Quality of cardiovascular care in an internal medicine resident clinic. $J$ Grad Med Educ 2010;2:467-73.

- Houlihan SJ, Simpson SH, Cave AJ, et al. Hypertension treatment and control rates: chart review in an academic family medicine clinic. Can Fam Physician 2009;55:735-41. 
These results may not apply to residents in other specialties or residents in other countries where the training is different. Future research should focus on identifying the barriers that currently exist to prevent physicians and patients from prioritising hypertension at every clinic visit. Research is needed to formulate interventions that circumvent these barriers in a constantly changing healthcare environment with limited resources.

In 2012, the CDC released a guide for cardiovascular care, advocating team-based care, shared ownership of patients, and better electronic panel management. ${ }^{11}$ This approach addresses many of the issues identified as possible barriers in this study. With teambased care, medical assistants and nurses who measure blood pressure would have the opportunity to bring abnormal readings directly to the attention of busy residents to ensure that findings are not overlooked. Shared ownership of patients between multiple residents would minimise the practice of perpetually deferring hypertension management to another physician, and improve continuity of care. Finally, more sophisticated electronic panel management would catch patients whose elevated blood pressure continues to go unaddressed visit after visit. Further research will be necessary to evaluate the effectiveness of these interventions on improving the quality of hypertension management in a residency setting.

Contributors PS analysed the data, drafted the paper and figures, and revised the paper. SYL designed the study, collected the data, and revised the paper.

Competing interests None.

Provenance and peer review Not commissioned; externally peer reviewed.

\section{REFERENCES}

1 CDC. Vital signs: high blood pressure is out of control for too many Americans, telebriefing. 4 September, 2012. http://www.cdc.gov/media/releases/2012/t0904_ hypertension.html (accessed 20 Jun 2013).

2 CDC. Vital signs: prevalence, treatment, and control of hypertension-United States, 1999-2002 and 2005-2008. MMWR 2011;60:103-8.

3 Chobanian AV, Bakris GL, Black HR, et al. Seventh report of the Joint National Committee on prevention, detection, evaluation, and treatment of high blood pressure. Hypertension 2003;42:1206-52.

4 Heidenreich PA, Trogdon JG, Khavjou OA, et al. Forecasting the future of cardiovascular disease in the United States: a policy statement from the American Heart Association. Circulation 2011;123:933-44.

5 Roger VL, Go AS, Lloyd-Jones DM, et al. Heart disease and stroke statistics-2012 update: a report from the American Heart Association. Circulation 2012;125:e2-20.

$6 \mathrm{Gu}$ Q, Dillon CF, Burt VL, et al. Association of hypertension treatment and control with all-cause and cardiovascular disease mortality among US adults with hypertension. Am J Hypertens 2010;23:38-45.

7 CDC. National Health and Nutrition Examination Survey: surveys and data collection systems (2010 data). Atlanta, GA: US Department of Health and Human Services, CDC, National Center for Health Statistics; 2012. Available at http://www.cdc.gov/ nchs/nhanes.htm (accessed 20 June 2013).

8 CDC. Vital signs: awareness and treatment of uncontrolled hypertension among adults-United States, 2003-2010. MMWR 2012;61:703-9.

9 Masterson E, Patel P, Kuo Y, et al. Quality of cardiovascular care in an internal medicine resident clinic. J Grad Med Educ 2010;2:467-73.

10 Houlihan SJ, Simpson SH, Cave AJ, et al. Hypertension treatment and control rates: chart review in an academic family medicine clinic. Can Fam Physician 2009:55:735-41.

11 Community Preventive Services Task Force. Guide to community preventive servicescardiovascular disease prevention and control: team-based care to improve blood pressure control. Atlanta, GA: US Department of Health and Human Services, CDC, 2012. http:// www.thecommunityguide.org/cvd/teambasedcare.html (accessed 20 Jun 2013). 